\title{
Trouble for cluster parameter estimation from blind SZ surveys?
}

\author{
N. Aghanim ${ }^{1}$, S. H. Hansen ${ }^{2}$, and G. Lagache ${ }^{1}$ \\ 1 IAS - CNRS, Bâtiment 121, Université Paris Sud, 91405 Orsay, France \\ e-mail: aghanim@ias.u-psud.fr \\ 2 University of Zurich, Winterthurerstrasse 190, 8057 Zurich, Switzerland
}

Received 24 February 2004 / Accepted 9 March 2005

\begin{abstract}
The Sunyaev-Zel'dovich (SZ) effect of galaxy clusters is a tool to measure three quantities: Compton parameter, electron temperature, and cluster peculiar velocity. However, a major problem is non-removed contamination by astrophysical sources that emit in the SZ frequencies. This includes interstellar dust emission, infra-red (IR) galaxies, and radio sources in addition to primary Cosmic Microwave Background (CMB) anisotropies. The three former contaminations induce systematic shifts in the three SZ parameters. In this study, we carefully estimated, both for a large beam experiment (namely Planck Surveyor) and a small beam experiment (ACT-like), the systematic errors that result if a fraction of the expected levels of emission from dust, IR galaxies, and radio sources remains non-removed. We found that the interstellar dust emission is not a major contaminant for the SZ measurement. Unfortunately, the IR and radio source-induced systematic errors may be extremely large. In particular the intra-cluster temperature and peculiar velocity will be determined inaccurately for Planck and ACT-like experiments, if only the frequency dependences are used for the cleaning. The Compton parameter is also affected by the astrophysical contaminations. The systematic errors in this case were a factor of 2 to 5 times larger than the expected statistical error-bar for Planck. For the ACT-like experiment, the statistical error-bars were larger than in the case of Planck by a factor of about 5 , and therefore the systematic shifts remain within about $50 \%$ of the statistical errors. We have thus shown that the systematic errors due to contaminating astrophysical emissions can be significantly larger than the statistical errors, which implies that future SZ surveys aiming at measuring cluster temperatures and peculiar velocities will not be able to do so on their own without including additional information like cluster shapes or follow-up observations.
\end{abstract}

Key words. cosmology: cosmic microwave background - galaxies: cluster: general

\section{Introduction}

The well-studied Sunyaev-Zel'dovich (SZ) effect (Sunyaev \& Zeldovich 1972; Sunyaev \& Zel'dovich 1980) has been observed towards a few tens of known galaxy clusters (see reviews by Carlstrom et al. 2002; and Birkinshaw 1999). It is a powerful tool for cosmological and cluster studies, and when combined with other observations (X-rays, optical, lensing) the SZ effect allows us to measure cosmological parameters such as the Hubble constant and matter density in the universe $\Omega_{\mathrm{m}}$ (e.g. Myers et al. 1997; Grego et al. 2001; Reese et al. 2002; Battistelli et al. 2003). The SZ effect is independent of redshift and is therefore an excellent tracer of large scale structure formation and evolution. This property has inspired groups to propose blind SZ surveys and investigate the way they can probe the cosmological parameters (e.g. Bartlett \& Silk 1994; Barbosa et al. 1996; Holder et al. 2000; Da Silva et al. 2000; Kneissl et al. 2001; Xue \& Wu 2001). As a consequence, several SZ experiments are either planned, under construction, or already observing. Some of these experiments are interferometric arrays like AMIBA (Lo et al. 2001), AMI
(Kneissl et al. 2001), SZA ${ }^{1}$ (Mohr et al. 2002). Others are single dish multi-frequency instruments like Planck surveyor $^{2}, \mathrm{SPT}^{3}, \mathrm{ACT}^{4}$, OLIMPO (Masi et al. 2003), SUZIE-II ${ }^{5}$, ACBAR $^{6}$, MITO $^{7}$.

The SZ effect can also be used to characterise the galaxy clusters themselves. Pointecouteau et al. (1998) proposed measuring the intra-cluster gas temperature from the relativistic corrections to the SZ. This is a particularly important issue in the context of future SZ surveys for which X-ray counterparts will not be easily available and since X-ray temperatures have not been measured for all clusters. This is because $\mathrm{X}$-ray temperature determination is expensive, and one cannot expect that all the many clusters to be observed in future SZ surveys will have X-ray temperature determination. The SZ effect can also be used to measure cluster radial peculiar

\footnotetext{
//astro.uchicago.edu/sza/

2 //www.rssd.esa.int/Planck/

//astro.uchicago.edu/spt

4 //www.hep.upenn.edu/ ${ }^{\sim}$ angelica/act/act.html

5 //www.stanford.edu/ schurch/suzie_instrument.html

6 //cosmology.berkeley.edu/group/swlh/acbar/

7 //oberon.roma1.infn.it/mito/
} 
velocities as suggested by Sunyaev \& Zel'dovich (1980). This provides a new distance free measurement of the cluster velocities, which is very interesting in view of future SZ surveys. However this needs measurement of the intra-cluster temperature. So far, it is only for a handful of known rich clusters that attempts have been made to extract the intra-cluster gas temperature (Hansen et al. 2002) or to extract the peculiar radial velocities (Holzapfel et al. 1997; Lamarre et al. 1998; Benson et al. 2003). It is fair to say that only upper limits have been obtained, and an actual temperature or peculiar velocity determination has still to be made.

The SZ effect is a potentially powerful cosmological probe; however, it is not free of contamination. As a matter of fact, SZ measurements are contaminated by other astrophysical sources that are mainly of two types: (i) due to our galaxy (free-free, synchrotron and dust emission from the milky way) or (ii) due to extra-galactic point sources (radio and infra-red (IR) galaxies), and even (iii) due to the Cosmic Microwave background (CMB) itself. The kinetic SZ and primary CMB fluctuations have the same frequency dependence, and therefore a separation of the CMB signal and the kinetic effect will only be possible through the relativistic corrections to the kinetic effect. This would require both very high sensitivity (of the order $0.1 \mu \mathrm{K}$ ) and additional observing frequencies at the extrema of the relativistic corrections to the kinetic effect (near 200 and $500 \mathrm{GHz}$ ) and at its cross-over (near $300 \mathrm{GHz}$ ). The effect of the primary CMB anisotropies is well known (see for example Haehnelt \& Tegmark 1996, and Aghanim et al. 1997). It induces a further uncertainty to the peculiar velocity and limits its accuracy. This effect intervenes mostly for large beam experiments like Planck, as small beam experiments are indeed less affected by the CMB which power is severely damped on a scale of about a few arcminutes.

These contaminations may be monitored in the context of pointed SZ observations (i.e. towards known clusters) or follow-up observations of likely clusters. However, systematic follow-up observations of all the clusters in the SZ survey will be very time-consuming. Ignoring contaminations modifies the effectiveness of a survey by the loss of certain clusters and the appearance of some other "fake" artificial clusters. For future surveys accurate knowledge of the completeness and reliability is thus crucial for extracting cosmological information. The question of survey selection function is being studied extensively for the Planck survey (White 2003; Geisbusch et al. 2005; Schafer et al. 2004); however, the systematic effect from the assumed cluster structures still remains to be studied carefully (Birkinshaw \& Lancaster 2004; Hansen 2004a). Contamination of SZ measurements by extra-galactic sources, especially radio sources, is not a new problem (see for example reviews by Rephaeli 1995b; Birkinshaw 1999). It can be due to radio emission of the galaxies in the cluster itself (Ledlow \& Owen 1996; Cooray et al. 1998; Lin et al. 2002) or to foreground galaxies. More specifically, the emission of radio sources can dilute the SZ signal. Holder (2002) estimated the expected dilution and its effect on the SZ power spectrum. IR dusty galaxies whose emission dominates at high frequencies may also contaminate SZ measurements. In particular, gravitational lensing of dusty galaxies causes enhancement of the confusion noise, which is likely to affect SZ observations of nearby clusters (Blain 1998). The effect of the point sources (both radio and dusty galaxies) have been recently revisited by White \& Majumdar (2004), who quantified it in terms of an equivalent noise and computed the associated power spectra. Also recently, Knox et al. (2004) investigated the effects of IR galaxy contamination on the statistical error-bars of the SZ parameters. Contamination of SZ clusters by radio sources at low frequencies that can be monitored by the interferometric arrays remains potentially a very important source of errors for single dish experiments. On the other hand, multi-frequency observations with single dish experiments should help in solving the problem of contamination.

In this study, we have focused on one category of SZ instruments planned for SZ surveying, namely the single-dish multi-frequency experiments. The SZ number counts that will be provided to us by these surveys will certainly probe and constrain the cosmological parameters. However, such instruments are theoretically able not only to measure the SZ effect amplitude for each detected cluster (through the Compton parameter) but also its radial peculiar velocity and its gas temperature. We explored the capabilities of these future SZ surveys in terms of measuring the three cluster SZ parameters: Compton parameter, intra-cluster gas temperature, and radial peculiar velocity independently of other observations. In a previous work (Aghanim et al. 2003), we investigated the effects of the cluster parameter degeneracies of the above-mentioned cluster SZ parameters in terms of error-bars. Here we were interested in the effects of the different sources of contamination on the measurement of these parameters. The contamination did not affect the statistical error-bars strongly.

The main question we are seeking to answer in this article is: how big are the systematic errors due to non-removable contamination? We show that the systematic errors are very important. They are by far the dominant source of trouble for the future single-dish SZ blind surveys when these surveys are used alone to estimate cluster parameters of unresolved clusters. We start by presenting our models for the major astrophysical contaminants in Sect. 2, and we then discuss the SZ parameter extraction technique and the sample of galaxy clusters used in our study in Sect. 3. In Sects. 4 and 5, we present the results for the three SZ experiments under consideration in our study, and discuss the results in Sect. 6. We finally offer our conclusions in Sect. 7.

\section{Modeling the contaminants}

The contaminations strongly depend on the observing frequency. For galactic foregrounds the frequency dependance is given by the spectral energy distribution of the contaminants. For extra-galactic sources the frequency dependance results from the combination of the redshift evolution of the luminosity function with the spectral energy distribution of the sources. Moreover, the contamination levels, especially of the galactic foregrounds, also depend on the observed region of the sky and on the spatial distribution of the signal (e.g. the power spectrum of dust emission). Multi-frequency observations should help in reducing the contamination level through component separation, but we expect some contamination of the 
Table 1. Level of contaminations (in Jy/sr) for Planck. The first two lines are for the dust emission fluctuations in the $40 \%$ cleanest region of the sky, assuming a $k^{-3}$ spectrum when $30 \%$ and $100 \%$ of the fluctuations remain unremoved. The second two lines are for the CIB fluctuations. We computed the case where the IR galaxies are Poisson distributed and the case where we also take the correlations between sources into account. In this case, $\sigma_{\text {tot }}^{\mathrm{CIB}}=\sqrt{5 \times \sigma_{\text {Poisson }}^{2}}$. The last line is for the fluctuations due to the unresolved radio, while resolved radio sources represent $12 \%$ to $15 \%$ of the background.

\begin{tabular}{ccccccccc}
\hline \hline Frequency $(\mathrm{GHz})$ & 30 & 44 & 70 & 100 & 143 & 217 & 353 & 545 \\
\hline 30\% dust fluctuations & - & - & 3.96 & 13.09 & 33.76 & 144.69 & 704.14 & 2218.54 \\
100\% dust fluctuations & - & - & 13.22 & 43.65 & 112.53 & 482.29 & 2347.15 & 7395.14 \\
\hline Poisson CIB fluctuations & - & - & 311.27 & 583.41 & 1445.88 & 4426.90 & 15279.8 & 39082.8 \\
Poisson+correlated CIB fluctuations & - & - & 696.02 & 1304.54 & 3233.09 & 9898.85 & 34166.7 & 87391.8 \\
\hline Radio source fluctuations & 1246.7 & 1729.3 & 2830.4 & 3210.0 & 3204.5 & 3598.3 & - & - \\
\hline
\end{tabular}

measurements to remain. This is rendered even more critical due to the rather large uncertainties in the emission models used to describe the contaminating sources.

In order to address the question of statistical and systematic errors on the cluster SZ parameter extraction due to nonremovable contaminations, we computed the level of contamination of each source for a set of pre-defined frequencies. We based our study on an ensemble of planned SZ instruments which thus define our choice of frequencies. We distinguished between a large beam ( $\sim 5$ arcmin at best) experiment like Planck, and small beam ( $\sim 2$ arcmin) experiments ACTlike. For the Planck instrument, we excluded from our analysis the $857 \mathrm{GHz}$ channel which is totally dominated by IR emission from dust in the inter-stellar medium (ISM) and in high redshift galaxies. The ACT-like experiment had three observing frequencies 145,225 , and $265 \mathrm{GHz}$. We assumed an optimistic sensitivity of $2 \mu \mathrm{K}$ for each channel and a beam of 1.7 arcmin at $145 \mathrm{GHz}$, along with beam-size scaling as $1 /$ frequency.

\subsection{Dust in the ISM}

Thermal emission from dust particles in the ISM is an important source of radiation in the IR and submm domains $(v \geq$ $100 \mathrm{GHz}$ ). It thus contaminates the measurement of the SZ effect at high frequencies. We therefore did not compute its contribution to the 30 and $44 \mathrm{GHz}$ channels of Planck. Dust emission is usually modeled by a modified blackbody spectrum: $S_{v}^{\text {dust }}=\tau \times B_{v}\left(T_{\mathrm{d}}\right)$ where $B_{v}\left(T_{\mathrm{d}}\right)$ is the blackbody spectrum, $T_{\mathrm{d}}$ the dust temperature, and $\tau$ is the emissivity. The emissivity is proportional $\nu^{\beta}$ where $\beta$ is the spectral index. The values for $\beta$ and $T_{\mathrm{d}}$ are not unique but vary from dense clouds to diffuse gas. In the diffuse part of the sky $\left(N_{\mathrm{HI}} \leq 5 \times 10^{20}\right.$ atoms $\left./ \mathrm{cm}^{2}\right)$ the dust emission spectrum from the far-IR to the submillimeter does not spatially vary (Boulanger et al. 1996; Finkbeiner et al. 1999; Lagache 2003). Because of the difficulty of modeling dust emission properly, we instead computed the observed dust emission spectrum as in Lagache (2003) from a combination of COBE and WMAP measurements. We derived the mean spectrum of the dust emission correlated with $\mathrm{HI}$ in the diffuse part of the sky for $|b|>30^{\circ}$. This spectrum, which represents the far-IR to mm emission for $N_{\mathrm{HI}}=10^{20}$ atoms $/ \mathrm{cm}^{2}$, was used as a reference. The colors, i.e. the shape, of this spectrum are in very good agreement with Finkbeiner et al. (1999).
For the dust emission spatial distribution, our analysis relied on the power spectrum measured in $\mathrm{HI}$ (Miville-Deschênes et al. 2002) and in far-IR (Gautier et al. 1992; Miville-Deschênes et al. 2004), which is a steep power law spectrum with no break. We thus used the same power spectrum $\propto k^{-3}$ that describes the dust distribution over a very large range of angular scales.

To evaluate the fluctuation level of dust emission in the case of SZ experiments, we computed the dust emission power spectrum (in $\mathrm{Jy}^{2} / \mathrm{sr}$ ) at each observing frequency using the following expression:

$P(k)=4.8 \times 10^{5} \times\left(N_{\mathrm{HI}} / 10^{20}\right)^{2.1} \times(k / 0.01)^{-3} \times\left(B_{\lambda} / B_{100}\right)^{2}$

where $k$ is in $\operatorname{arcmin}^{-1}, N_{\mathrm{HI}}$ the $\mathrm{HI}$ column density in atoms $/ \mathrm{cm}^{-2}$, and the $B_{\lambda} / B_{100}$ the color given at each observing wavelength $\lambda=h v / c$ (in $\mu \mathrm{m}$ ). In our calculations, we used the column density associated with the cleanest $40 \%$ of the sky that corresponds to $N_{\mathrm{HI}} \sim 2.31 \times 10^{20}$ atoms $/ \mathrm{cm}^{2}$. We finally computed the fluctuations of the dust emission in the beam of the considered experiments which are given by $\sigma^{2}=\int P(k) 2 \pi k \mathrm{~d} k \mathrm{Jy}^{2} / \mathrm{sr}^{2}$. For a $k^{-3}$ power spectrum, the previous integral was dominated by the lower limit of $k$, which is taken to be equal to $1 / F W H M$.

The dust fluctuations cannot be removed and perfectly corrected for. In Tables 1 and 2 we show the level of contamination from dust fluctuations for Planck and for the ACT-like experiment for two cases: (i) where $30 \%$ of the fluctuations (i.e. $0.3 \sigma$ ) remain unremoved; and (ii) where $100 \%$ remains. This $30 \%$ is the realistic level of contamination when independent tracers of dust emission (such as $\mathrm{HI}$ ) are used to remove the dust contribution. The latter case (100\%) is displayed to show how the maximum contribution affects the results. In both cases (30 and $100 \%$ fluctuations), the contamination is computed for the $40 \%$ cleanest region of the sky in terms of gas column density, as found outside the galactic plane and in bright molecular clouds.

\subsection{Infra-red or "dusty" galaxies}

Star-forming galaxies not only emit in the near-IR, optical, and UV domains, but also in the far-IR and submm. This emission is associated with the radiation that is absorbed and re-emitted by the dust in galaxies. For local galaxies, up to one third of 
Table 2. Level of contaminations in Jy/sr for the ACT experiment. The first two lines are for the dust emission fluctuations in the $40 \%$ cleanest region of the sky when $30 \%$ and $100 \%$ of the fluctuations remain unremoved. The second two lines are for fluctuations of the $50 \%$ unresolved IR galaxies, when they are Poisson distributed and where we also take the correlations into account. In this case, $\sigma_{\text {tot }}^{\mathrm{CIB}}=\sqrt{5 \times \sigma_{\text {Poisson }}^{2}}$. The last line represents the fluctuations due to unresolved radio sources when $50 \%$ of the background is resolved.

\begin{tabular}{cccc}
\hline \hline Frequency (GHz) & 145 & 225 & 265 \\
\hline 30\% Dust fluctuations & 19.65 & 67.44 & 128.62 \\
100\% Dust fluctuations & 89.17 & 224.79 & 428.74 \\
\hline Poisson CIB fluctuations & 1225.19 & 5430.84 & 10877.9 \\
Poisson+Correlated CIB fluctuations & 2739.61 & 12143.7 & 24323.7 \\
\hline Radio sources fluctuations & 2265.42 & 2873.19 & 2821.53 \\
\hline
\end{tabular}

the output emission lies in the far-IR domain. Star-forming galaxies can have much larger fractions of their emitted radiation in the far-IR (up to 90\%). These galaxies are called "IR galaxies" in the following. The total emission from unresolved and/or faint IR galaxies is responsible for the Cosmic Infra-red Background (CIB) (Puget et al. 1996), which contains as much power as the optical and UV backgrounds. The fluctuations of the CIB that are now detected (Lagache et al. 2000; Matsuhara et al. 2000; Miville-Deschênes et al. 2002; Kiss et al. 2001) are one of the major contaminants of the SZ measurements at high frequencies $(\geq 100 \mathrm{GHz})$. As in the dust case, we will not compute the contribution at the 30 and $44 \mathrm{GHz}$ channels of Planck.

In order to evaluate the level of contamination by fluctuations of the CIB, i.e. unresolved dusty galaxies, we used the model of Lagache et al. (2003, 2004), which provided us with the number counts of IR galaxies at each frequency of the SZ experiments (Planck and ACT-like). We first estimated the IR background emission from the dusty galaxies by integrating the number counts below a given detection limit $S_{\mathrm{lim}}^{\mathrm{IR}}$. We then computed the rms fluctuations of the CIB due to unresolved sources in the beam of the SZ instruments. For this step, we considered two cases, one where the sources follow a Poisson distribution and a second where the correlation between sources is added to the shot noise. Song et al. (2003) have investigated the effect of correlations at high frequencies, and find it boosts the CIB shot noise fluctuations by a factor of 1.7 on average. Due to the lack of observational constraints or precise models for the correlations for all frequencies, we simply assumed that the correlated part of the CIB fluctuations is twice as large as the Poisson part for all frequencies and for all the experiments ${ }^{8}$. It is worth noting that we did not take possible enhancement by lensing into account. Our estimate of the CIB contribution is thus likely to be slightly underestimated especially for low redshift clusters.

We estimated the contribution of the CIB fluctuations to the SZ measurements in two cases: one case where only $1 \%$ of the dusty galaxies are resolved, which is the case predicted for Planck surveyor (Lagache et al. 2003). In the second case for the ACT-like experiment, we arbitrarily assumed $50 \%$ of the CIB is in resolved IR galaxies. The level of fluctuations are given for Planck and ACT-like in Tables 1 and 2, respectively.

\footnotetext{
${ }^{8}$ We have taken this factor 2 from the modeling of the clustering of the CIB anisotropies of Knox et al. (2001).
}

\subsection{Radio sources}

The radio emission of radio sources is dominated by synchrotron emission from relativistic electrons spiraling around magnetic fields in the sources. The synchrotron spectrum follows a power law $v^{\alpha}$, with $-1<\alpha<-0.5$. However, other types of sources exist such as those with "inverted spectra" (with $\alpha>0$ ). In general, the spectra are not simple power laws with fixed indexes (Herbig \& Readhead 1992), but have curved spectra instead; moreover some of the radio sources may be variable. It is therefore very hard to extrapolate their fluxes from one frequency to another. Additionally, radio sources are well catalogued at low frequencies thanks to NVSS (Condon et al. 1998) and FIRST (White et al. 1997), but there are no allsky surveys of extra-galactic sources at frequencies $>5 \mathrm{GHz}$ apart from the WMAP observations at $41 \mathrm{GHz}$ (Bennett et al. 2003). As a result, properties of the radio sources at high frequencies are poorly known. In the context of CMB observations, several studies have aimed at predicting the radio source counts at frequencies higher than $5 \mathrm{GHz}$ from known sources (Toffolatti et al. 1998; Sokasian et al. 2001). These studies allow us, in particular, to assess the level of contamination due to radio sources at the frequencies of interest for the $\mathrm{CMB}$, where they are the dominant contamination between 30 and $217 \mathrm{GHz}$. However, one has to keep in mind that extrapolations of radio spectra from $5 \mathrm{GHz}$ to high frequencies is quite a difficult task.

In our study, we compute the level of contamination of unresolved radio sources per frequency by integrating the source counts below a flux limit, $S_{\text {lim }}^{\text {radio }}$, determined for each frequency. Assuming Poisson distribution for the sources, we then evaluate the rms fluctuations due to unresolved radio sources in the beam of each SZ experiment (Planck and ACT-like). We did not take the effect of the correlation of radio sources into account since it is rather weak. It is worth noting in the case of Planck, in particular, that the source fluctuations increase with frequency although radio sources are getting fainter. This is due to the decrease of the beam-size with frequency from $33 \mathrm{arcmin}$ at $30 \mathrm{GHz}$ to $5.5 \mathrm{arcmin}$ at $217 \mathrm{GHz}$. In practice, we use the radio-source counts that were kindly provided by L. Toffolatti and are based on the model of Toffolatti et al. (1998). This model was found to be in quite good agreement with recent observations of radio sources by WMAP at $41 \mathrm{GHz}$. It is thus likely to provide us with a good prediction of the radio counts at higher frequencies. As mentioned above, to estimate 
the level of contamination from unresolved sources we need the flux limit at each frequency for the detection of radio sources. In the case of Planck frequencies, Vielva et al. (2001) provided us with the flux limits $S_{\text {lim }}^{\text {radio }}$ above which the radio sources are detected. They were computed using simulated observed sky taking all galactic and extra-galactic emissions into account, together with instrumental noise. These flux limits correspond to $5 \sigma$ detections. As a consequence, the residual fluctuations computed in Table 1 are likely to be overestimated.

Using the flux limits of Vielva et al. (2001) ranging from $S_{\mathrm{lim}}^{\text {radio }}=540 \mathrm{mJy}$ (at $30 \mathrm{GHz}$ ) to $S_{\mathrm{lim}}^{\text {radio }}=240 \mathrm{mJy}$ (at $217 \mathrm{GHz}$ ), we found that about $12 \%$ to $15 \%$ of the radio background was resolved into individual sources (detected at $5 \sigma$ ) by Planck. The levels of contamination are summarised in Table 1. For ACT-like, we investigated the effect of unresolved radio sources on the cluster parameter measurement by assuming arbitrarily and optimistically that $50 \%$ of the radio background is resolved into sources. The numbers are given in Table 2 . Contrary to what is stated in Knox et al. (2004), the radio source contamination always dominates over the fluctuations from IR galaxies up to $217 \mathrm{GHz}$ when the latter are Poisson distributed, and up to $143 \mathrm{GHz}$ when we take the correlations of IR galaxies into account. We did not compute the contributions at 353 and $545 \mathrm{GHz}$ for Planck since they become quite subdominant.

\subsection{Synchrotron and free-free}

The synchrotron emission in our galaxy is due to the emission of free electrons spiraling around the lines of magnetic field. This emission is difficult to model because of poor knowledge of the galactic magnetic field itself. Synchrotron emission is expected to dominate at low frequencies $(<80 \mathrm{GHz})$. Bennett et al. (2003) argue that this emission in fact dominates over all other CMB foregrounds in the WMAP frequency bands. But at high galactic latitudes in the diffuse sky (where the SZ observations will be conducted), Lagache (2003) show that the WMAP emission is dominated by the so-called "anomalous microwave emission" associated with the small interstellar transiently heated dust grains. This component was included in our measured dust spectrum.

We did not include the free-free emission that is, in the diffuse sky at high latitudes, much smaller than the amplitude of the anomalous microwave component. We also ignore a possible contamination from ultra relativistic electrons (Ensslin \& Kaiser 2000), which is at best marginally detectable with future SZ surveys (Ensslin \& Hansen 2004).

\section{SZ parameters}

The SZ effect is traditionally separated into two components according to the origin of the scattering of the same electrons

$$
\begin{aligned}
\frac{\Delta I(x)}{I_{0}} & =\Delta I_{\text {thermal }}\left(x, y, T_{\mathrm{e}}\right)+\Delta I_{\text {kinetic }}\left(x, \tau, v_{\mathrm{p}}\right) \\
& =y\left(g(x)+\delta_{\mathrm{T}}\left(x, T_{\mathrm{e}}\right)\right)-\beta \tau h(x),
\end{aligned}
$$

with $x=h v / k T_{\mathrm{cmb}}$ and $I_{0}=2\left(k T_{\mathrm{cmb}}\right)^{3} /(h c)^{2}$ where $T_{\mathrm{cmb}}=$ $2.725 \mathrm{~K}$. The first term on the rhs of Eq. (1) is the thermal distortion (Sunyaev \& Zeldovich 1972) with the nonrelativistic spectral shape

$g(x)=\frac{x^{4} \mathrm{e}^{x}}{\left(\mathrm{e}^{x}-1\right)^{2}}\left(x \frac{\mathrm{e}^{x}+1}{\mathrm{e}^{x}-1}-4\right)$,

and the magnitude is given by the Compton parameter

$y=\frac{\sigma_{\mathrm{T}}}{m_{\mathrm{e}} c^{2}} \int \mathrm{d} l n_{\mathrm{e}} k T_{\mathrm{e}}$,

where $m_{\mathrm{e}}$ and $n_{\mathrm{e}}$ are masses and number density of the electrons, $\sigma_{\mathrm{T}}$ is the Thomson cross section, and the integral is along the line of sight. For non-relativistic electrons one has $\delta_{\mathrm{T}}\left(x, T_{\mathrm{e}}\right)=0$, but for hot clusters the relativistic electrons will slightly modify the thermal SZ effect (Wright 1979). The spectral shape of $\delta_{\mathrm{T}}\left(x, T_{\mathrm{e}}\right)$ is easily calculated numerically (Rephaeli 1995a; Dolgov et al. 2001; Shimon \& Rephaeli 2003; Sandoval-Villalbazo \& Garcia-Colin 2003; Itoh $\&$ Nozawa 2004). For the Planck frequencies this is easily implemented using the simple fits presented in Diego et al. (2003).

The kinetic distortions (Sunyaev \& Zel'dovich 1980) in the last term of Eq. (1) have the spectral shape

$h(x)=\frac{x^{4} \mathrm{e}^{x}}{\left(\mathrm{e}^{x}-1\right)^{2}}$,

and the magnitude depends on $\beta=v_{\mathrm{p}} / c$, the average line-ofsight streaming velocity of the thermal gas (positive if the gas is approaching the observer), and the Thomson optical depth

$\tau=\sigma_{\mathrm{T}} \int \mathrm{d} l n_{\mathrm{e}}$

The minor thermal corrections to the kinetic effect are negligible with existing sensitivity. Thus, when the intra-cluster gas can be assumed isothermal one has $y=\tau k T_{\mathrm{e}} /\left(m_{\mathrm{e}} c^{2}\right)$, while for non-isothermal clusters things are slightly more complicated (Hansen 2004c).

Given the different spectral signatures of $g(x), h(x)$, and $\delta_{\mathrm{T}}\left(x, T_{\mathrm{e}}\right)$, it is straightforward to separate the physical variables $y, v_{\mathrm{p}}$, and $T_{\mathrm{e}}$ from sensitive multi-frequency observations. This separation is easily understood as follows. To a good approximation the magnitude of the SZ effect gives the $y$ parameter, which is measured at the maximum of $g(x)$ (near 129 or $370 \mathrm{GHz}$ ). The velocity times optical depth, $\beta \tau$, is measured at the zero-point of $g(x)$ (near $217 \mathrm{GHz}$ ). Finally, electron temperature $T_{\mathrm{e}}$ is measured using the zero point and maxima of $\delta_{\mathrm{T}}$ (near 190, 330, $475 \mathrm{GHz}$ ). Hence, under the assumption of isothermality, these observables can be combined to provide the physical parameters $y, v_{\mathrm{p}}$, and $T_{\mathrm{e}}$. It is thus clear that with at least 3 well-placed observing frequencies one can separate all 3 physical variables by using only the $\mathrm{SZ}$ observations. The first marginal determination of all $3 \mathrm{SZ}$ parameters was made for the hot cluster A2163 (Hansen et al. 2002) using data from SUZIE (Holzapfel et al. 1997) and BIMA/OVRO (LaRoque et al. 2002). However, as was shown in Aghanim et al. (2003) additional observing frequencies are needed to break the intrinsic degeneracies between the SZ cluster parameters. 


\subsection{The practical parameter extraction}

In order to find the SZ parameters for a given cluster we modified the publicly available SZ-parameter extraction code SASZ. For a given "SZ observation" this code fast and accurately determines the central value for the $3 \mathrm{SZ}$ parameters $\left(y, v_{\mathrm{p}}\right.$, and $T_{\mathrm{e}}$ ), and corresponding $1 \sigma$ error-bars (Hansen 2004b). These error-bars reflect solely the statistical error-bars due to the observational sensitivity (which for Planck or ACT-like is on the order of a few $\mu \mathrm{K}$ for each channel). This parameter extraction code is based on the stochastic method of simulated annealing, and we chose the cooling scheme of SASZ in such a way that the central values of the $3 \mathrm{SZ}$ parameters of all the clusters in our sample were determined to better than $0.1 \%$ accuracy.

\subsection{The galaxy cluster sample}

To finally realistically answer the question of how big the systematic errors due to non-removable contamination are, one must first of all consider a large sample of clusters. We simulated clusters in bins of mass and redshift, using an extended Press-Schechter formalism that provides realistic temperatures, Compton parameters, and peculiar velocities (for details see Aghanim et al. 2001, 2003). In our study, we considered clusters with redshifts between 0.2 and 3 . Within this range of redshifts, not all clusters fill the beam of the instrument (i.e. $10 R_{\mathrm{c}}=$ beam - size, with $R_{\mathrm{c}}$ the cluster core radius), which sets a limit to the cluster mass range. We illustrate this point in the case of Planck, for which we set a conservative value of 9 arcmin for the effective beam-size of the instrument for cluster observations. In this case, all clusters with total masses between $5 . \times 10^{13}$ and $10^{16}$ solar masses and redshifts $z>0.5$ fill the beam. However, at lower redshifts $z=0.2(0.3$ and 0.4$)$ only clusters with masses above 1.4. $\times 10^{15}\left(4 . \times 10^{15}\right.$ and $\left.8 . \times 10^{15}\right)$ solar masses fulfill this condition. Specifically we consider 500 hot clusters with temperatures in the range $3.5 \mathrm{keV}<T_{\mathrm{e}}<$ $7.5 \mathrm{keV}$, central Compton parameter $-4.7<\log (y)<-3.7$, and peculiar velocity $-1600 \mathrm{~km} \mathrm{~s}^{-1}<v_{\mathrm{p}}<1600 \mathrm{~km} \mathrm{~s}^{-1}$. For each cluster we thus have the true values, $y_{\text {true }}, T_{\text {true }}$, and $v_{\text {true }}$, from which one can calculate the true SZ signal, $\Delta I_{\mathrm{SZ}}$.

Under the assumption that all contaminations have been removed completely, the total signal is equal to the SZ signal (ignoring the noise), $\Delta I_{\text {total }}=\Delta I_{\mathrm{SZ}}$. Thereby, one can import the total signal to SASZ, which then finds the best fit parameters, $y_{\text {derived }}, T_{\text {derived }}$, and $v_{\text {derived }}$. For all the 500 clusters in our sample the central values of the parameters have been determined to better than $0.1 \%$ accuracy.

Now, the interesting question is which central values are found for the SZ parameters when the contaminations have not been removed completely. For instance, if the dust has not been removed completely, then there will be an additional signal, $\Delta I_{\text {dust }}$. In that way the total signal is $\Delta I_{\text {total }}=\Delta I_{\mathrm{SZ}}+\Delta I_{\text {dust }}$. Believing this signal is purely from SZ will induce a systematic error, $y_{\text {derived }}=y_{\text {true }}+\delta y_{\text {systematic }}$, but it will not significantly change the statistical error due to instrumental sensitivity. We explicitly compared the statistical error-bars due to the instrumental sensitivity for slightly different cluster parameters, and found the effect on the statistical error-bars very moderate (a few percent). We will now address the question of how big these systematic errors are. We show that these systematic errors for future planned SZ surveys can be significantly larger than the statistical errors due to instrumental sensitivity.

\section{Planck surveyor case}

As the first example we consider the all-sky Planck survey. The expected error-bars on the SZ parameters from Planck observations without taking the contaminations into account have already been studied (Aghanim et al. 2003). Due to its wide frequency range, earlier predictions estimated that the Compton parameter should be easily measured and that the cluster peculiar velocity should be very marginally estimated with an error of about 500-700 $\mathrm{km} \mathrm{s}^{-1}$. In particular the importance of non-Gaussian error-bars was discussed in detail in Aghanim et al. (2003). In our study, we considered a simplification to the real error-bars. Instead of having different upper and lower error-bars, we simply took the largest of the two; that is, for all observables we consider $T_{-\delta T_{\text {low }}}^{+\delta T_{\text {up }}}$, with $\delta T=\delta T_{\text {up }}=\delta T_{\text {low }}=$ $\max \left(\delta T_{\text {up }}, \delta T_{\text {low }}\right)$.

In Fig. 1 we present the expected Planck statistical errorbars on $y$ within this simplification. In the idealised case where no contamination is taken into account, Compton parameter $y$ is found to an accuracy of $1 \%$ for the brightest clusters with $y=10^{-3.7}$ and to $10 \%$ for the dimmer clusters in our sample with $y=10^{-4.7}$. The temperature is determined at best with a few $\mathrm{keV}$, and more typically with 5-10 keV, statistical errorbars. The size of the statistical error-bars on $v_{\mathrm{p}}$ depends very much on the actual value of $v_{\mathrm{p}}$, and goes from few hundred $\mathrm{km} / \mathrm{s}$, for the best case, to $2000-3000 \mathrm{~km} \mathrm{~s}^{-1}$ for the worst. It is worth noting that for our implementation of the extended Press-Schechter formalism (Sect. 3.2) we binned the data in cluster mass and redshift. This results in a quantization of the values (e.g. $\log (y))$ and gives the impression of a binning in the figures. This has no impact on the results, since all we need is a sample of cluster parameters that roughly span the ranges expected in a standard cosmology.

We next added the various non-removed contaminations to the signal to investigate their effects on the measured parameters. More explicitly, with a pure SZ signal, $\Delta I_{\text {measured }}=\Delta I_{\mathrm{sz}}$, one would derive the "true" SZ parameters, whereas with a contaminated signal, $\Delta I_{\mathrm{obs}}=\Delta I_{\mathrm{sZ}}+\Delta I_{\text {contam }}$ one finds incorrect derived SZ parameters. Namely, the contamination causes systematic shifts. In order to quantify the importance of such systematic shifts, we compared them to the statistical errorbars, given above. We compared the value of e.g. $T_{\text {derived }}-T_{\text {true }}$ to $\delta T$. Since the statistical error-bars from above are conservative, then our findings represent lower limits to the magnitude of the systematic shift on the SZ parameters due to contamination. Our sample contains 500 clusters, and we considered (for $T_{\mathrm{e}}$, for example) the rms value, over the sample, of the ratio $T_{\text {derived }}-T_{\text {true }}$ to $\delta T$, which is given by:

$\epsilon_{T}=\frac{1}{N^{1 / 2}}\left(\sum_{i=1}^{N}\left(\frac{T_{i}^{\text {derived }}-T_{i}^{\text {true }}}{\delta T_{i}}\right)^{2}\right)^{1 / 2}$ 


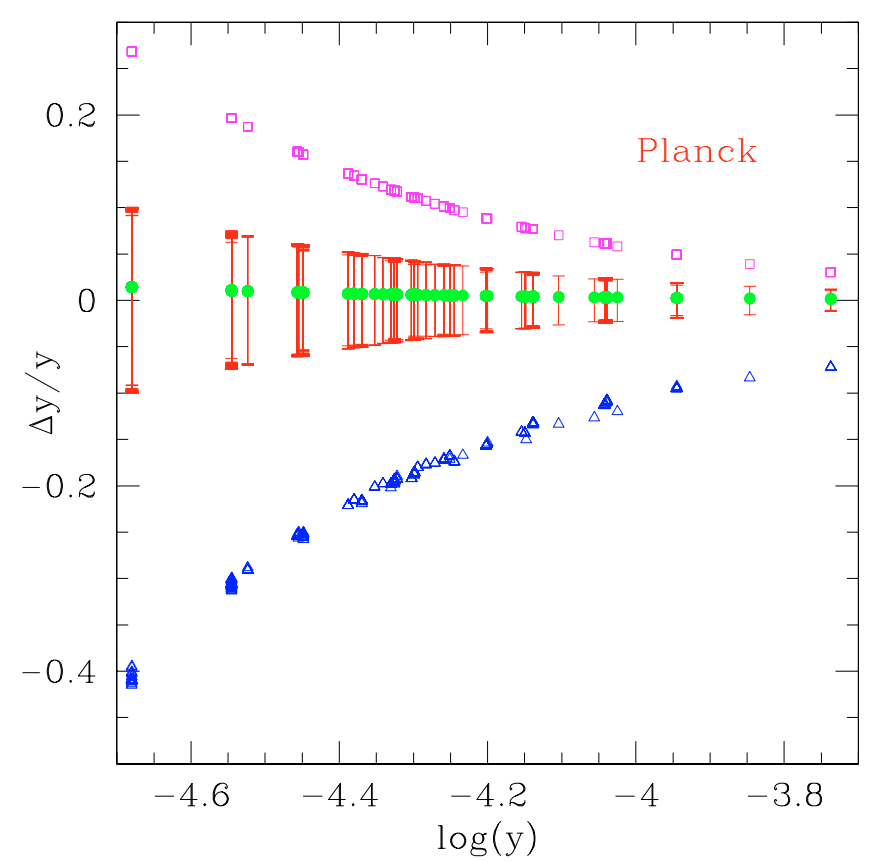

Fig. 1. The expected error-bars (red) on the Compton parameter for the Planck experiment, without contamination. The open triangles (blue) show the effect due to non-removed radio contamination. The open squares (purple) are for correlated IR galaxies, and the filled circles (green) are when none of the dust emission fluctuations have been removed.

We present the results in Figs. 1-3 where we plot the central values of the derived parameters when adding the contaminations. In the case of interstellar dust on the $40 \%$ cleanest part of the sky (filled circles), the systematic shifts are found to be quite small when $70 \%$ of the fluctuations are removed. The effect is small even when no dust fluctuations are removed. We found $\epsilon_{y}($ dust $) \approx \epsilon_{v}($ dust $) \approx 0.15$, and the temperature is only very marginally affected. We can therefore conclude that the contamination from the fluctuations of interstellar dust emission will not be a major problem for estimating the three SZ parameters $\left(y, T_{\mathrm{e}}\right.$, and $\left.v_{\mathrm{p}}\right)$ in the Planck case.

The situation is completely different when we considered the contamination due to the fluctuations of the IR background (CIB). In that case, we found $\epsilon_{y}(\mathrm{CIB}) \approx 2.5, \epsilon_{v}(\mathrm{CIB}) \sim 2$, and found minor temperature shift. We also checked the effect of contamination by CIB Poisson fluctuations only. We found that the rms ratio between systematic and statistical errors are about 0.5 for $y$ and $v_{\mathrm{p}}$ and negligible for the temperature.

The situation is even worse when we consider contamination from fluctuations due to the unresolved and unremoved radio sources. In this case, we found a very large contamination of the Compton parameter $\epsilon_{y}($ radio $) \approx 5, \epsilon_{T}$ (radio $) \sim$ $\epsilon_{v}$ (radio) $\sim 1$. As mentioned above, the level of radio fluctuations is likely to be overestimated since it was computed using flux limits for $5 \sigma$ detections. We redid our analysis, assuming $20 \%$ of the radio sources to be resolved (less conservative than the $12-15 \%$ of Vielva et al. 2001), in which case the Compton parameter are less affected by the radio contamination, $\epsilon_{y}($ radio $) \approx 3$. When dust, $\mathrm{CIB}$, and radio fluctuations are added there is a slight improvement of the determination of the

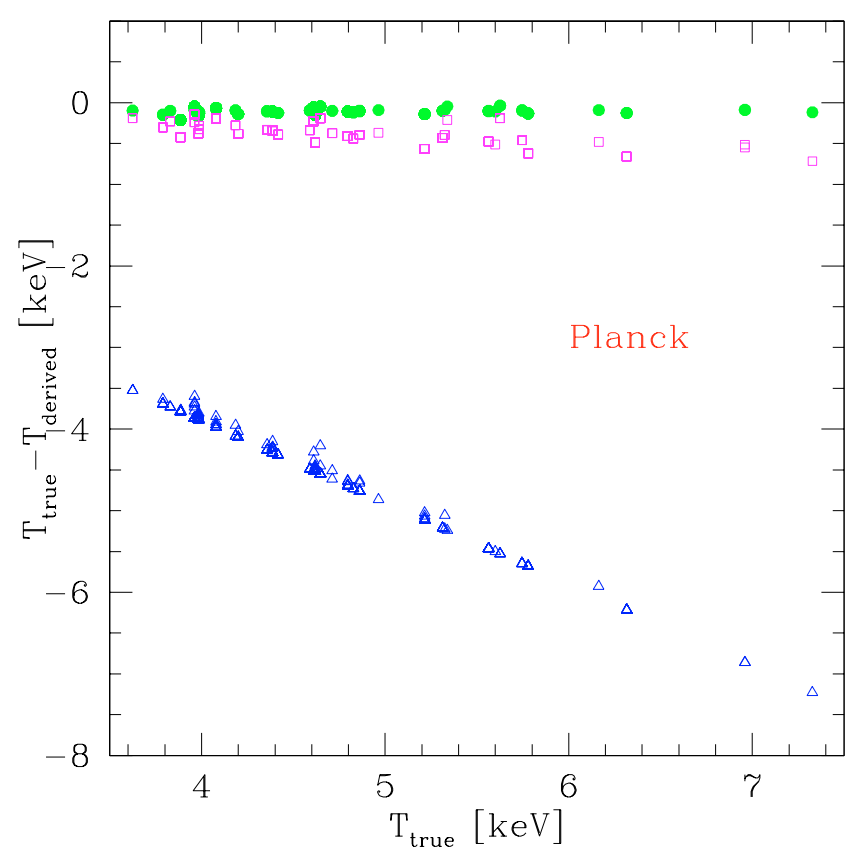

Fig. 2. Derived electron temperature in $\mathrm{keV}$. Open triangles (blue) are for radio contamination, open squares (purple) are for Poisson+correlated IR galaxies, and filled circles (green) are for dust fluctuations. Statistical error-bars (of the order few to ten $\mathrm{keV}$ ) are not shown in order to avoid confusion.

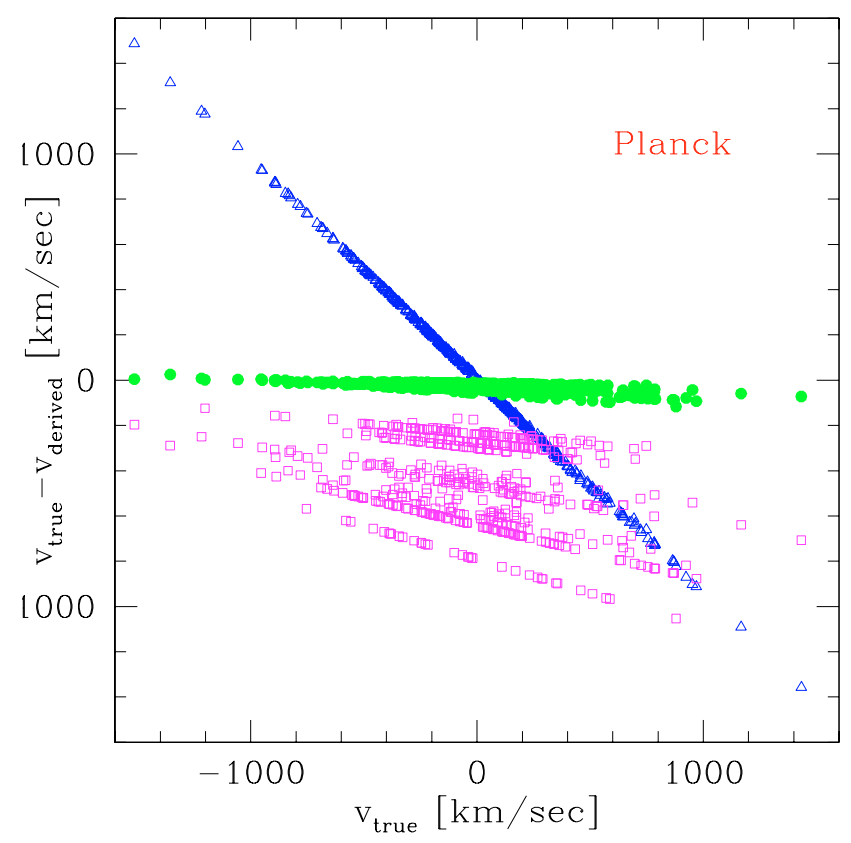

Fig. 3. Upper limit to the expected effect of contamination in the derived peculiar velocity in $\mathrm{km} \mathrm{s}^{-1}$. Open triangles (blue) are for radio contamination, open squares (purple) for Poisson+correlated IR galaxies, and filled circles (green) for dust fluctuation. Statistical errorbars (on the order of a few 100 to a few $1000 \mathrm{~km} \mathrm{~s}^{-1}$ ) are not shown, in order to avoid confusion.

Compton parameter. We find $\epsilon_{y}=1.7$; however, this does not improve determination of the temperature and velocities. We emphasize that this is an accidental partial cancellation, which cannot be counted on in general. 


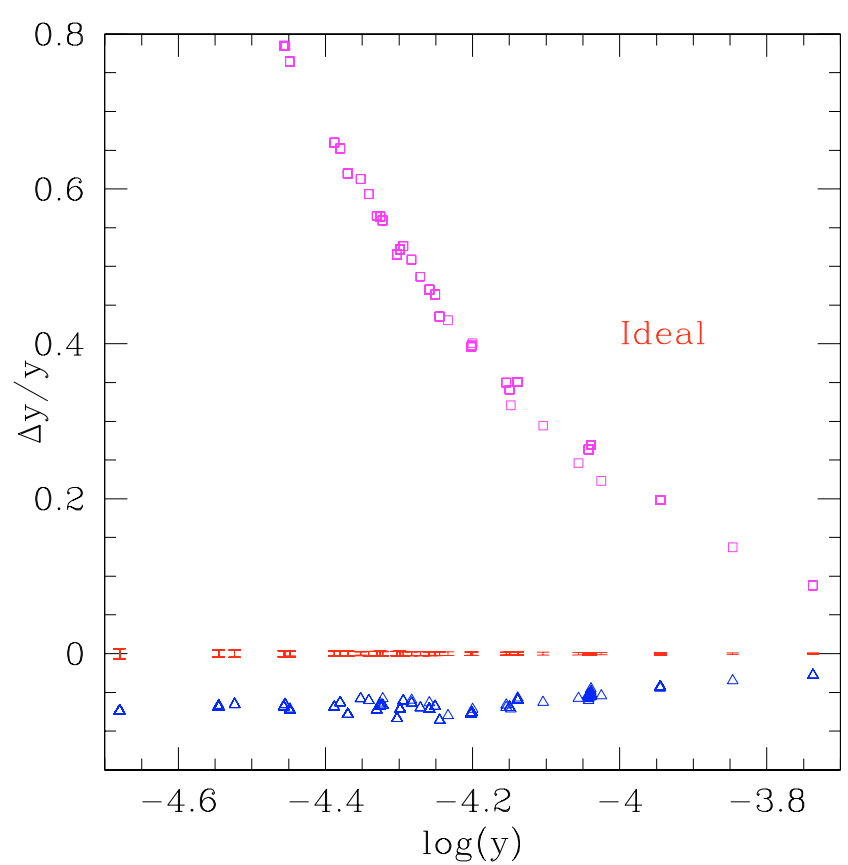

Fig. 4. Compton parameter for an ideal experiment with 4 observing frequencies at $100,150,217$, and $270 \mathrm{GHz}$, and with $0.1 \mu \mathrm{K}$ sensitivity. Open triangles (blue) for radio contamination; open squares (purple) are for Poisson+correlated IR galaxies. The very small statistical error-bars without contamination are shown in red. Systematic shift from dust fluctuations is very small and is not shown as it would obscure the statistical error-bars.

The effects of the different contaminations on the SZ parameters are qualitatively easy to understand. They are illustrated with an ideal low noise experiment $(0.1 \mu \mathrm{K})$ observing at 100,150, 217 and $270 \mathrm{GHz}$ with about 1 arcmin resolution. First, a contamination from radio sources gives a positive contribution at low frequencies, where the dominating $y$ parameter is determined, leading to a lower value for $y$ (see triangles in Fig. 4). Now, with this lower value for $y$, the high frequency signal seems too high, which can only be counterbalanced with a very low temperature (see triangles in Fig. 5). Finally, the velocity term reads as $v_{\mathrm{p}} / T_{\mathrm{e}}$, so with a very low temperature, $v_{\mathrm{p}}$ is also forced to be very small. In reality, this leads approximately to $T_{\mathrm{e}} \approx v_{\mathrm{p}} \approx 0$.

Both IR galaxies and dust emission give a positive contribution at high frequencies. The latter can be compensated for in 3 ways, either 1) y parameter is larger (see squares in Fig. 4); 2) temperature is lower (see squares in Fig. 5); or 3) peculiar velocity $v_{\mathrm{p}}$ is slightly more negative. We see from Figs. 1-3, that the actual result is a rather complicated adjustment between these 3 possibilities. The lines of squares in Fig. 3 appear because of our quantization in cluster masses and have no physical relevance. The systematic shift in peculiar velocity for a randomly observed cluster will be somewhere within the range of the symbols. The contaminating signal was added at each frequency for each cluster, rather than a population of contaminating signals; and therefore each cluster is represented by only one symbol. We thus present an upper limit to the expected effect of contamination, and the effect for a given cluster can be smaller.

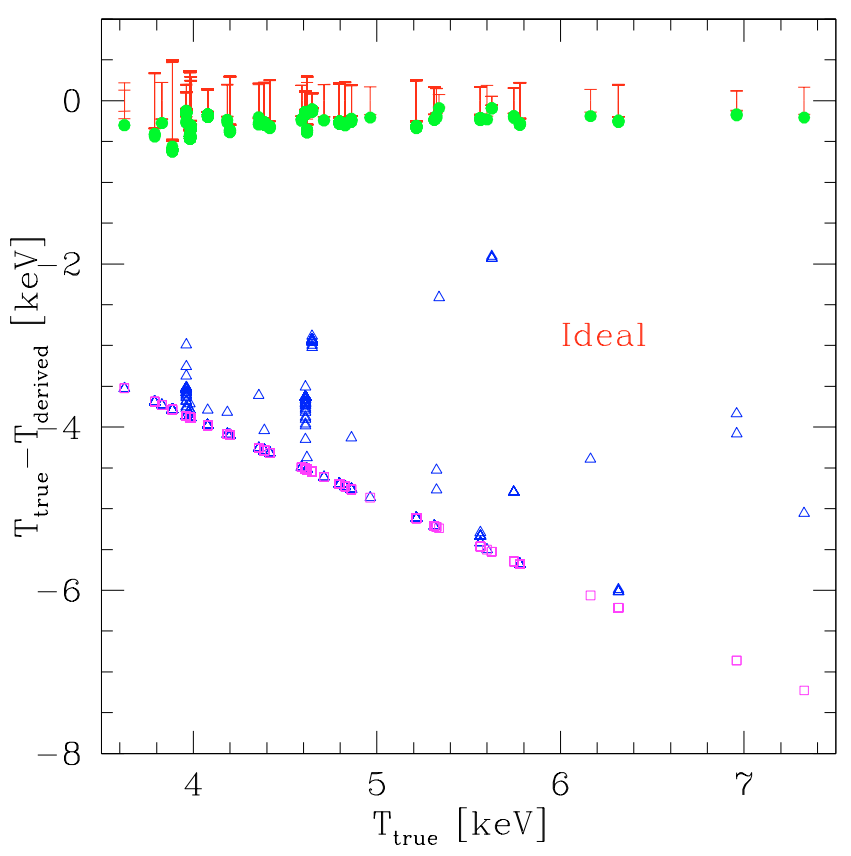

Fig. 5. Derived electron temperature in $\mathrm{keV}$ for an ideal experiment. The very small statistical error-bars are shown in red. Open triangles (blue) are radio contamination, open squares (purple) are Poisson+correlated IR galaxies, and filled circles (green) are for dust fluctuations.

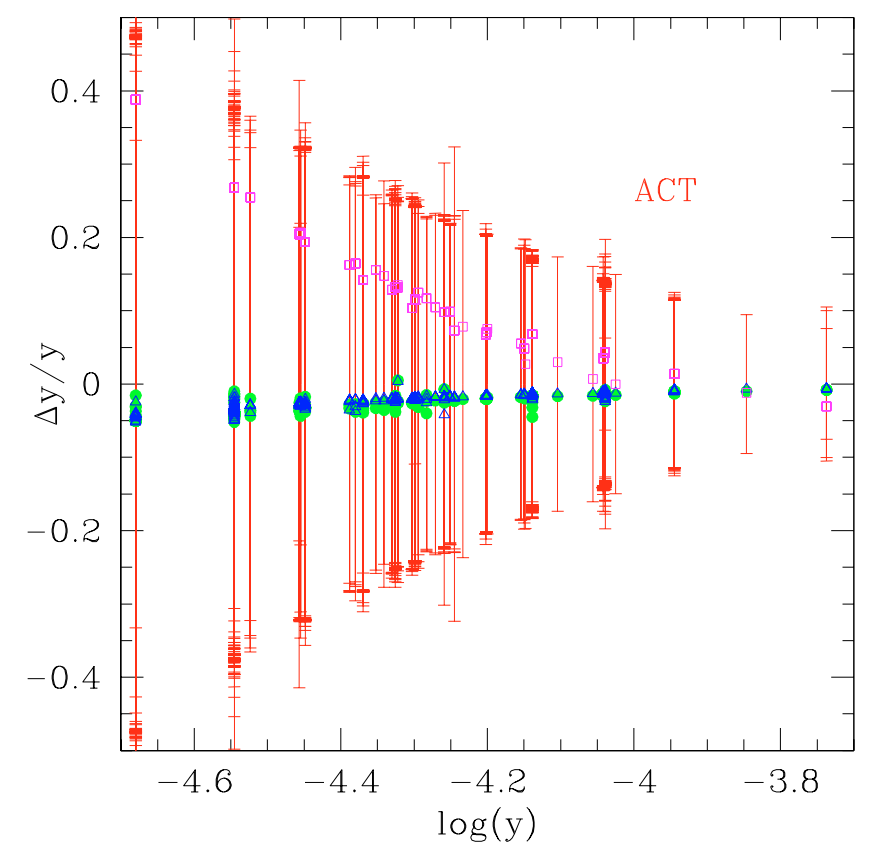

Fig. 6. Compton parameter for ACT-like survey. The statistical errorbars without contamination are shown in red. Open triangles (blue) are radio contamination, open squares (purple) Poisson+correlated IR galaxies, and filled circles (green) dust fluctuations.

In the Planck case, one also has to take the contamination from the CMB primary anisotropies themselves into account. This contribution and its effect were already studied and evaluated to an error of a few hundred $\mathrm{km} \mathrm{s}^{-1}$ (Haehnelt \& Tegmark 1996; Aghanim et al. 1997). In particular, this was done in the context of a Wiener filtering analysis for the component 


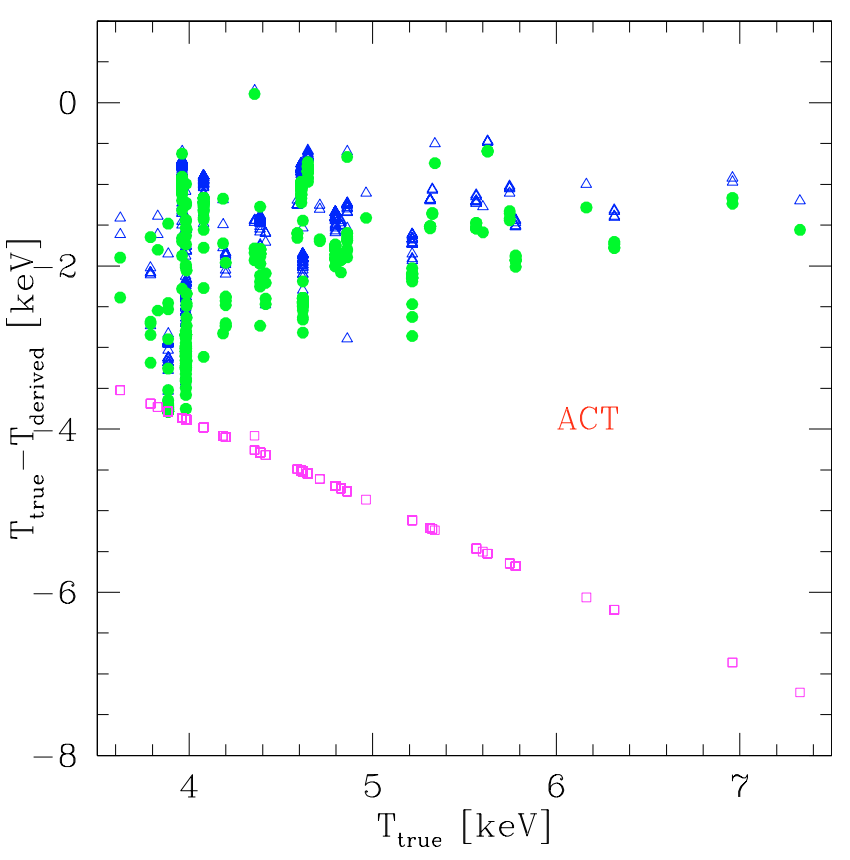

Fig. 7. Derived electron temperature in keV. Open triangles (blue) are radio contamination, open squares (purple) are Poisson+correlated IR galaxies, and filled circles (green) are dust fluctuations. Statistical error-bars (the smallest about $5 \mathrm{keV}$, but more typical error-bars are around $10-20 \mathrm{keV}$ ) are not shown in order to avoid confusion.

separation taking a detailed simulation of the millimeter and submillimeter sky into account. The contamination by the primary CMB anisotropies slightly shifts the central values of the parameters without modifying the error-bars significantly. We therefore chose not to display it in the figures.

\section{Future ACT-like SZ surveys}

A number of future experiments are planned to perform SZ surveys of a hundred to a few thousand square degrees. They expect to detect several thousand SZ clusters. We investigated the effects of the contaminations for those future ACT-like surveys that have a narrower frequency coverage but a smaller beamsize than the Planck surveyor.

To be specific, we assumed that the future ACT-like experiment has three observing frequencies 145,225 , and $265 \mathrm{GHz}$, with angular resolution of 1.7 arcmin at $145 \mathrm{GHz}$ (with beamsize scaling as 1 /frequency), and sensitivity of $2 \mu \mathrm{K}$ for each channel. As a result of the smaller frequency coverage of such experiments, it will be more complicated for them to disentangle the SZ effect from other sources which emit at the same frequencies. They will have a smaller beam-size of $\sim 1.5$ arcmin as compared to the 5 arcmin Planck beam. This makes the contribution from interstellar dust smaller since it is dominated by large scale fluctuations. The CMB contamination will be quite negligible due to the sharp cut-off at small angular scales. On the other hand, those contributions due to fluctuations of CIB or unresolved radio sources will be larger than in the Planck case. However, more sources will be resolved; we thus set the fraction of resolved sources to an optimistic value of 50\%. Moreover for ACT-like experiments, the number

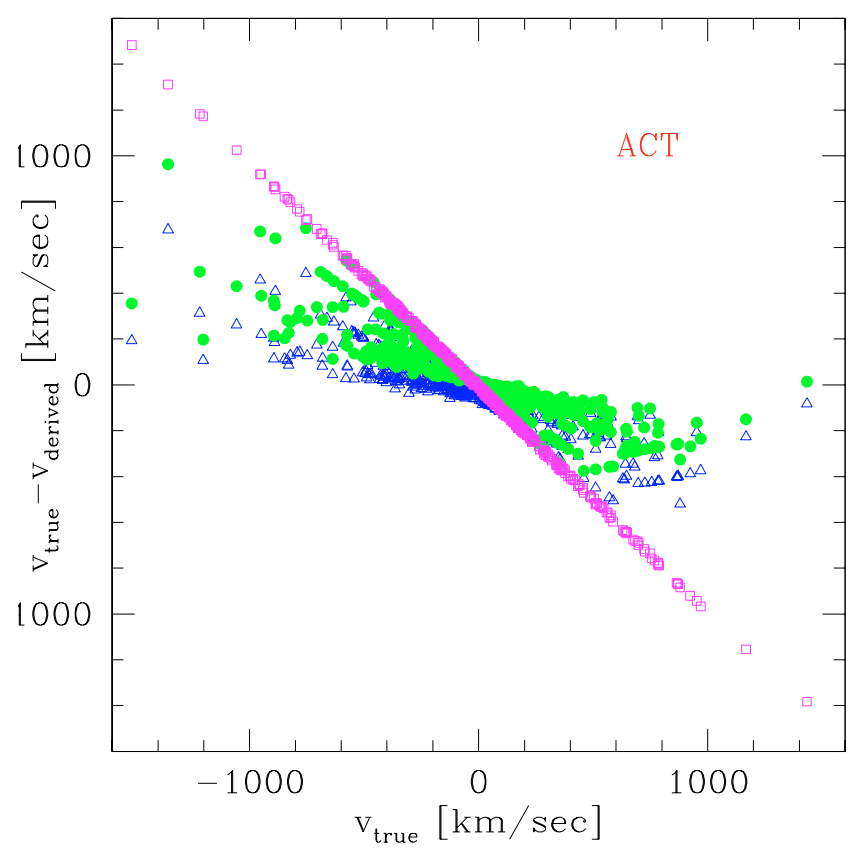

Fig. 8. Upper limit to the expected effect of contamination in the derived peculiar velocity in $\mathrm{km} \mathrm{s}^{-1}$. Open triangles (blue) are radio contamination, open squares (purple) Poisson+correlated IR galaxies, and filled circles (green) dust fluctuations. Statistical error-bars (the smallest are about $500 \mathrm{~km} \mathrm{~s}^{-1}$, but more typical error-bars are $2000-6000 \mathrm{~km} \mathrm{~s}^{-1}$ ) are not shown, in order to avoid confusion.

of clusters that fill the beam is much smaller than for Planck. Up to $z=0.4$ all clusters with masses between $5 . \times 10^{13}$ and $10^{16}$ solar masses will be resolved, i.e. exceed the beam size. The use of adapted filtering techniques like those proposed in Schafer et al. (2004) should help in reducing the contamination in such clusters. At higher redshifts up to $z \sim 0.7$, clusters with masses below $2 . \times 10^{14}$ fill the beam but they mostly represent the clusters close to the detection limit. It is only at high redshifts $(z>1)$ that more massive clusters $\left(1 . \times 10^{15}\right.$ solar masses) fulfill our condition on the beam.

The few observing channels and moderate sensitivity of an ACT-like experiment makes determination of temperature and peculiar velocity very difficult. Statistical error-bars are typically much larger than the central values themselves. For temperatures the smallest statistical error-bars are about $5 \mathrm{keV}$, and more typical error-bars are $10-20 \mathrm{keV}$. For peculiar velocity the smallest error-bars are several hundred $\mathrm{km} \mathrm{s}^{-1}$, and more typically it is several thousands $\mathrm{km} \mathrm{s}^{-1}$. Therefore, even if a given contamination implies a complete failure in determining the central values, it still remains within the statistical error-bar.

It is slightly different for the Compton parameter, for which expected error-bars are between 10 and 50\%. Both dust and radio contaminations will have a negligible impact $\left(\epsilon_{y}(\right.$ dust $) \sim$ $\epsilon_{y}($ radio $\left.) \sim 0.1\right)$. Only IR galaxies will have a noticeable effect with systematic shifts in $y$ of up to $40 \%$ in the worst cases (and $\epsilon_{y}(\mathrm{CIB})=0.4$ ). It has been suggested (Knox et al. 2004) that including a $30 \mathrm{GHz}$ channel (from complementary observations) will improve all these statistical error-bars (in particular for $y$ ), but as we saw above, this channel will be significantly 
contaminated by radio sources, which again makes $y$ too small (and pushes both $T_{\mathrm{e}}$ and $v_{\mathrm{p}}$ towards zero).

\section{Requirements for SZ observations}

Our results for Planck show that the dust contamination on the $40 \%$ cleanest part of the sky is not a major source of error for the SZ parameter estimation even in the most pessimistic case when all the dust fluctuations remain unremoved. On the other hand, the fluctuating background from the IR or radio unresolved sources is an important issue. For the CIB fluctuations, we show that the Poisson fluctuations do not introduce major systematic shifts in contrast to the case where we take the correlations into account. The fluctuations from unresolved radio sources are the main sources of errors in the SZ parameters. This contamination dominates over the CIB fluctuations up to 143 or $217 \mathrm{GHz}$ when only Poisson or Poisson+correlated CIB fluctuations are present, respectively.

In order to reduce the systematic effects on the SZ parameters, we need to reduce the contaminations. A rather reasonable demand must be that for each contaminant the induced systematic error must be smaller than the statistical error, and hopefully much smaller. If we require that the ratio of systematic to statistical errors be smaller than $1 / 2$ for each contaminant, then we find that for Planck the radio contamination must be reduced by a factor of 13 , and the contamination from CIB must be reduced by a factor of about 5 . For the radio sources, this is attainable when $\sim 60 \%$ of the radio background is resolved in individual sources, as compared to the $12-15 \%$ given by the present detection limits for Planck. This corresponds to a flux limit of about $2 \mathrm{mJy}$ at each frequency contaminated by radio emission (from $3 \mathrm{mJy}$ at $44 \mathrm{GHz}$ to $1.5 \mathrm{mJy}$ at $217 \mathrm{GHz}$ ). For the CIB, reduction of the contamination by a factor 5 corresponds to resolving about $60 \%$ of the background with flux limits ranging between 0.67 and $0.008 \mathrm{mJy}$ from higher to lower frequencies. It is worth noting that due to confusion limit, at present only $50 \%$ of the CIB is resolved in individual sources in blank field surveys at $350 \mathrm{GHz}$ (Lagache et al. 2003). This is achieved by the SCUBA instrument in small fields (less than a few square degrees) and with a 10 arcsec resolution.

In all cases (for Planck but also for ACT-like), the requirement to perform SZ parameter estimation from SZ observations is to observe on regions of the sky where the CIB and radio contamination levels can be pinned down. This is only possible by resorting to dedicated follow-up surveys, in radio and IR at higher resolution, to complement the SZ observations. However, such follow-up observations, in particular in radio, have to be done at frequencies close to the SZ measurements to avoid large errors from the extrapolation of spectral energy distributions (Ricci et al. 2003). Both large fields (a significant fraction of the SZ surveys themselves) and angular resolutions (less than 3 arcsec typically) to beat confusion limits are mandatory. Future interferometers (e.g. ALMA) will have the required angular resolution but will not be able to cover large enough areas. More specifically, for ALMA to resolve $50 \%$ of the CIB in individual sources (number used for ACT-like experiments) at $230 \mathrm{GHz}$, the detection limit is $0.1 \mathrm{mJy}(5 \sigma)$. In such conditions, 138 days are needed to survey $1 \mathrm{deg}^{2}$. It is even worse for resolving $80 \%$ of the CIB; 96 days are needed for $10 \mathrm{arcmin}^{2}$ with a detection limit of $0.02 \mathrm{mJy}(5 \sigma)$. For Planck, only the brightest sources need to be removed, which can be achieved with rather short observations.

Contamination by radio and IR point sources will be less of a problem for the interferometric SZ experiments, which naturally have better resolutions to monitor point sources.

\section{Discussion}

In our study, we have shown that the measurement of SZ parameters $\left(y, T_{\mathrm{e}}\right.$, and $\left.v_{\mathrm{p}}\right)$, which is theoretically feasible with multi-frequency observations of the clusters, is complicated in practice by the presence of unremoved or non-removable contaminations. In some cases, it is even made impossible. Even for very accurate observations of the SZ effect, i.e. with small error-bars, the contaminations induce systematic shifts in parameter values. These systematic shifts can be extremely large, particularly in the case of two sources of contaminations: fluctuations from unresolved radio sources and fluctuations of the cosmic infrared background. Such contaminations were recently addressed by Knox et al. (2004). However, these authors estimated the statistical errors on the SZ parameters rather than the induced shifts in the derived values. They were also studied by White \& Majumdar (2004). Rather than studying their effects on the SZ parameters, these authors estimated the level of noise introduced the IR and radio sources in SZ surveys, and compared it to the predicted SZ power spectrum.

For the CIB and radio fluctuations, the systematic shift dominates the source of errors. Furthermore, the results we obtained can become even worse, since we chose neither to take the amplification of the CIB and radio emission by lensing effects into account (Blain 1998), nor to include the effects of correlations for the radio sources and central radio source in clusters. Note that for ACT-like surveys we took an arbitrarily optimistic level of $50 \%$ for the unresolved IR and radio backgrounds, which cannot be achieved due to the resolution and the frequency of the experiment.

The quantitative results we obtained for the systematic shifts due to contamination by spurious sources of emission in the SZ frequencies are based on the hypothesis that the clusters under study fill the beam of the experiment. This will be the case for the major part of the Planck cluster catalogue; however, we do not expect this to be the case for ACT-like survey, for example, as the beam is much smaller $(\sim 1.5$ arcmin). Additional information provided by the spatial extension of the clusters helps in better constraining the optical depth $\tau$ and thus the Compton parameter itself. Such additional constraints will reduce the errors on the $\mathrm{SZ}$ parameter estimation for the next generation of experiments like ACT. They will not be as helpful in the case of Planck, where most of the clusters will fill the beam. Similarly, measurement of polarised emission from the clusters might help in better constraining $\tau$ and thus $y$, provided the clusters do not fill the beam of the instrument. However, the polarised signal is weak and the measurement quite difficult.

It seems from our results that the $\mathrm{SZ}$ observations alone will not provide us with the the three cluster parameters. If we specifically want to estimate the peculiar velocities, we are 
forced to do it on known clusters. It is only on known objects that we might expect to properly correct for the contaminations as was done e.g. in Lamarre et al. (1998) on cluster A2163. However, such a procedure will consume time and effort. Another possibility might be to use extra information from scaling relations and/or X-ray complementary observations. However, the use of X-ray observed scaling relations is unwise, partly because the weight from non-isothermal clusters is different between X-ray observations and SZ observations (e.g. Hansen 2004a), and the use of numerical scaling relations is still far from being sufficiently reliable due to the complication in simulating gas dynamics.

\section{Conclusion}

The future blind SZ surveys will be contaminated by astrophysical sources that can only be partly removed, such as interstellar dust emission, infra-red galaxies, and radio sources. Such non-removable contamination will induce a systematic shift in the derived SZ parameters. We have shown that IR and radio source-induced systematic errors may be extremely large, virtually removing the possibility of measuring peculiar velocity and cluster temperature if using purely the SZ observations. Also the systematic shift in the Compton parameter will be significantly larger than the expected statistical error-bars. Therefore these contaminations are potentially disastrous for future survey experiments, and must be considered very as very serious. On the other hand, the situation may be less desperate if the contamination levels are reduced by complementary follow-up surveys. As mentioned in Sect. 6, the radio followups should be planned at, or close to, the SZ frequencies and the radio source variability requires simultaneous monitoring. CIB fluctuations need much better resolution than what is accessible now. One must therefore be realistic about future SZ surveys, and consider them as excellent tools for identifying high redshift clusters and not as tools for measuring peculiar velocity or cluster temperature.

Acknowledgements. The authors wish to thank an anonymous referee for his/her comments and suggestions which helped us to improve this study. It is a pleasure to thank L. Toffolatti for providing us with the radio-source counts used in the present study. We also thank F.-X. Désert and J.-L. Puget for useful remarks. This project was partly supported by the CNRS grant ACI-Jeunes chercheurs "de la physique des hautes énergies à la cosmologie observationnelle : développement d'un groupe de cosmologie sur le campus d'Orsay". S.H.H. thanks the Tomalla foundation for support.

\section{References}

Aghanim, N., De Luca, A., Bouchet, F. R., Gispert, R., \& Puget, J.-L. 1997, A\&A, 325, 9

Aghanim, N., Górski, K. M., \& Puget, J.-L. 2001, A\&A, 374, 1

Aghanim, N., Hansen, S. H., Pastor, S., \& Semikoz, D. V. 2003, JCAP, 5,7

Barbosa, D., Bartlett, J. G., Blanchard, A., \& Oukbir, J. 1996, A\&A, 314, 13

Bartlett, J. G., \& Silk, J. 1994, ApJ, 423, 12
Battistelli, E. S., De Petris, M., Lamagna, L., et al. 2003, ApJ, 598, L75

Bennett, C. L., Hill, R. S., Hinshaw, G., et al. 2003, ApJS, 148, 97

Benson, B. A., Church, S. E., Ade, P. A. R., et al. 2003, ApJ, 592, 674

Birkinshaw, M. 1999, Phys. Rept., 310, 97

Birkinshaw, M., \& Lancaster, K. 2004 [arXiv:astro-ph/0410336]

Blain, A. W. 1998, MNRAS, 297, 502

Boulanger, F., Reach, W. T., Abergel, A., et al. 1996, A\&A, 315, L325

Carlstrom, J. E., Holder, G. P., \& Reese, E. D. 2002, ARA\&A, 40, 643

Condon, J. J., Cotton, W. D., Greisen, E. W., et al. 1998, AJ, 115, 1693

Cooray, A. R., Grego, L., Holzapfel, W. L., Joy, M., \& Carlstrom, J. E. 1998, AJ, 115, 1388

Da Silva, A. C., Barbosa, D., Liddle, A. R., \& Thomas, P. A. 2000, MNRAS, 317, 37

Diego, J. M., Hansen, S. H., \& Silk, J. 2003, MNRAS, 338, 796

Dolgov, A. D., Hansen, S. H., Pastor, S., \& Semikoz, D. V. 2001, ApJ, 554, 74

Ensslin, T. A., \& Kaiser, C. R. 2000, A\&A, 360, 417

Ensslin, T. A., \& Hansen, S. H. 2004

[arXiv:arXiv: astro-ph/0401337]

Finkbeiner, D. P., Davis, M., \& Schlegel, D. J. 1999, ApJ, 524, 867

Gautier, T. N. I., Boulanger, F., Perault, M., \& Puget, J. L. 1992, AJ, 103,1313

Geisbusch, J., Kneissl, R., \& Hobson, M. 2005, MNRAS, 360, 41

Grego, L., Carlstrom, J. E., Reese, E. D., et al. 2001, ApJ, 552, 2

Haehnelt, M. G., \& Tegmark, M. 1996, MNRAS, 279, 545

Hansen, S. H. 2004a [arXiv: astro-ph/0410004]

Hansen, S. H. 2004b, New Astron., 9, 279

Hansen, S. H. 2004c, MNRAS, 351, L5

Hansen, S. H., Pastor, S., \& Semikoz, D. V. 2002, ApJ, 573, L69

Herbig, T., \& Readhead, A. C. S. 1992, ApJS, 81, 83

Holder, G. P., Mohr, J. J., Carlstrom, J. E., Evrard, A. E., \& Leitch, E. M. 2000, ApJ, 544, 629

Holder, G. P. 2002, ApJ, 580, 36

Holzapfel, W. L., Ade, P. A. R., Church, S. E., et al. 1997, ApJ, 481, 35

Itoh, N., \& Nozawa, S. 2004, A\&A, 417, 827

Kiss, C., Abraham, P., Klaas, U., Juvela, M., \& Lemke, D. 2001, A\&A, 379,1161

Kneissl, R., Jones, M. E., Saunders, R., et al. 2001, MNRAS, 328, 783

Knox, L., Cooray, A., Eisenstein, D., \& Haiman, Z. 2001, ApJ, 550, 7

Knox, L., Holder, G., \& Church, S. 2004, ApJ, 612, 96

Lagache, G. 2003, A\&A, 405, 813

Lagache, G., Haffner, L. M., Reynolds, R. J., \& Tufte, S. L. 2000, A\&A, 354, 247

Lagache, G., Dole, H., \& Puget, J.-L. 2003, MNRAS, 338, 555

Lagache, G., Dole, H., Puget, J.-L., et al. 2004, ApJS, 154, 112

Lamarre, J.-M., Giard, M., Pointecouteau, E., et al. 1998, ApJ, 507, L5

LaRoque, S. J., Carlstrom, J. E., Reese, E. D., Holder, G. P., et al. 2002 [arXiv: astro-ph/0204134]

Ledlow, M. J., \& Owen, F. N. 1996, AJ, 112, 9

Lin, H., Chiueh, T., \& Wu, X.-P. 2002 [arXiv: arXiv: astro-ph/0202174]

Lo, K. Y., Chiueh, T. H., Martin, R. N., et al. 2001, 20th Texas Symposium on relativistic astrophysics, AIP Conf. Proc., 586, 938

Masi, S., Ade, P., De Bernardis, P., et al. 2003, Mem. S.A. It.,74, 96

Matsuhara, H., Kawara, K., Sato, Y., et al. 2000, A\&A, 361, 407

Miville-Deschênes, M.-A., Lagache, G., \& Puget, J.-L. 2002, A\&A, 393, 749

Miville-Deschênes, M.-A., Lagache, G., \& Puget, J.-L. 2004, in preparation 
Mohr, J. J., Carlstrom, J. E. and SZA scientific collaboration 2002, AMIBA 2001, ASP Conf. Proc., 257, 43

Myers, S. T., Baker, J. E., Readhead, A. C. S., Leitch, E. M., \& Herbig, T. 1997, ApJ, 485, 1

Pointecouteau, E., Giard, M., \& Barret, D. 1998, A\&A, 336, 44

Reese, E. D., Carlstrom, J. E., Joy, M., et al. 2002, ApJ, 581, 53

Rephaeli, Y. 1995a, ApJ, 445, 33

Rephaeli, Y. 1995b, ARA\&A, 33, 541

Ricci, R., Ekers, R. D., Staveley-Smith, L., et al. 2003, Maps of the Cosmos, International Astronomical Union, Symp. 216

Sandoval-Villalbazo, A., \& Garcia-Colin, L. S. 2003, Gen. Rel. Grav., 37,831

Schafer, B. M., Pfrommer, C., Hell, R. M., \& Bartelmann, M. 2004 [arXiv: astro-ph/0407090]

Shimon, M., \& Rephaeli, Y. 2003, New Astron., 9, 69

Sokasian, A., Gawiser, E., \& Smoot, G. F. 2001, ApJ, 562, 88
Song, Y. S., Cooray, A., Knox, L., \& Zaldarriaga, M. 2003, ApJ, 590, 664

Sunyaev, R. A., \& Zel'dovich, Ya. B. 1972, Comm. Astrophys. Space Phys., 4, 173

Sunyaev, R. A., \& Zel'dovich, Ya. B. 1980, ARA\&A, 18, 537

Toffolatti, L., Argueso Gomez, F., De Zotti, G., et al. 1998, MNRAS, 297, 117

Vielva, P., Martinez-Gonzalez, E., Cayon, L., et al. 2001, MNRAS, 326, 181

White, R. L., Becker, R. H., Gregg, M. D., et al. 1997, 191st A\&AS Meet., BAAS, 29, 1373

White, M. 2003, ApJ, 597, 650

White, M., \& Majumdar, S. 2004, ApJ, 602, 565

Wright, E. L. 1979, ApJ, 232, 348

Xue, Y. J., \& Wu, X. P. 2001, ApJ, 552, 452 